\title{
Häufig gebrauchte Abkürzungen
}

\section{A Liganden und Verbindungen}

\begin{tabular}{|c|c|}
\hline Ac & Acetyl, $\mathrm{CH}_{3} \mathrm{CO}$ \\
\hline acac & Acetylacetonat; 2,4 -Pentandionat, $\mathrm{CH}_{3} \mathrm{COCHCOCH}_{3}{ }^{-}$ \\
\hline acacH & Acetylaceton; 2,4-Pentandion \\
\hline $\mathrm{AcO}$ & Acetat \\
\hline ADP & Adenosindiphosphat \\
\hline ala & Alaninat(1-), $\mathrm{CH}_{3} \mathrm{CH}\left(\mathrm{NH}_{2}\right) \mathrm{COO}^{-}$ \\
\hline An & Actinoide (Elemente 90-103) \\
\hline Ar & Aryl \\
\hline $\mathrm{ArH}$ & Aren \\
\hline ATP & Adenosintriphosphat \\
\hline BAL & Britisches Antilewisit; 2,3-Dimercaptopropanol \\
\hline benzac & Benzoylacetat(1-) \\
\hline bgH & Biguanid, $\mathrm{H}_{2} \mathrm{NC}(\mathrm{NH}) \mathrm{NHC}(\mathrm{NH}) \mathrm{NH}_{2}$ \\
\hline bpy & $2,2^{\prime}$-Bipyridyl \\
\hline $\mathrm{Bu}$ & Butyl [ $n$-(normal); $i$-(iso); $s$-(sekundär); $t$-(tertiär) $]$ \\
\hline Chl & Chlorophyll \\
\hline $\mathrm{CoA}$ & Coenzym A \\
\hline $\operatorname{cod}$ & 1,5-Cyclooctadien, $\mathrm{C}_{8} \mathrm{H}_{12}$ \\
\hline $\cot$ & Cyclooctatetraen, $\mathrm{C}_{8} \mathrm{H}_{8}$ \\
\hline $\mathrm{cp}$ & Cyclopentadienyl-Radikal; Cyclopentadienyl-Anion \\
\hline crypt & Cryptand \\
\hline cy & Cyclohexyl \\
\hline Cys & Cystein \\
\hline Cyt & Cytochrom \\
\hline deta & trans-1,2-Diaminocyclohexantetraacetat(4-) \\
\hline DDT & 1,1,1-Trichlor-2,2-bis( $p$-chlorphenyl)ethan \\
\hline depe & 1,2-Bis(diethylphosphano)ethan; Ethylenbis(diethylphosphan) \\
\hline depm & 1,2-Bis(diethylphosphano)methan; Methylenbis(diethylphosphan) \\
\hline diars & $o$-Phenylenbis(dimethylarsan), $o$ - $\mathrm{C}_{6} \mathrm{H}_{4}\left(\mathrm{AsMe}_{2}\right)_{2}$ \\
\hline dien & Diethylentriamin, $\left(\mathrm{NH}_{2} \mathrm{CH}_{2} \mathrm{CH}_{2}\right)_{2} \mathrm{NH}$ \\
\hline diglyme & Diethylenglykoldimethylether, $\mathrm{H}_{3} \mathrm{CO}\left(\mathrm{CH}_{2} \mathrm{CH}_{2} \mathrm{O}\right)_{2} \mathrm{CH}_{3}$ \\
\hline diphos & Chelat-bildendes Bisphosphan (meist dppe) \\
\hline $\mathrm{dlm}$ & Diallylamin, $\left(\mathrm{CH}_{2}=\mathrm{CH}-\mathrm{CH}_{2}\right)_{2} \mathrm{NH}$ \\
\hline dma & Dimethylacetamid; (als Lsgm. meist DMA) \\
\hline dme & Dimethoxyethan; Ethylenglykoldimethylether (glyme) \\
\hline $\mathrm{dmf}$ & Dimethylformamid, $\mathrm{HC}(\mathrm{O}) \mathrm{N}\left(\mathrm{CH}_{3}\right)_{2}$; (als Lsgm. meist DMF) \\
\hline dmg & 2,3-Butandion-dioximat-Dianion \\
\hline dmgH & 2,3-Butandion-dioximat-Monoanion \\
\hline $\mathrm{dmgH}_{2}$ & 2,3-Butandion-dioxim (Dimethylglyoxim; Biacetyldioxim) \\
\hline dmpe & 1,2-Bis(dimethylphosphano)ethan; Ethylenbis(dimethylphosphan) \\
\hline dmso & Dimethylsulfoxid; (als Lsgm. meist DMSO) \\
\hline DNA & Desoxyribonucleinsäure \\
\hline dppe & 1,2-Bis(diphenylphosphano)ethan; Ethylenbis(diphenylphosphan) \\
\hline dppm & Bis(diphenylphosphano)methan; Methylenbis(diphenylphosphan) \\
\hline dsep & Diethyldiselenophosphat $(1-)$ \\
\hline $\mathrm{dtc}$ & Diethyldithiocarbamat(1-) \\
\hline dtp & Diethyldithiophosphat (1-) \\
\hline dtpa & Diethylentriaminpentaacetat $(5-)$ \\
\hline $\mathrm{E}$ & Element (i. a. Nichtmetall) \\
\hline
\end{tabular}


XXVI Häufig gebrauchte Abkürzungen

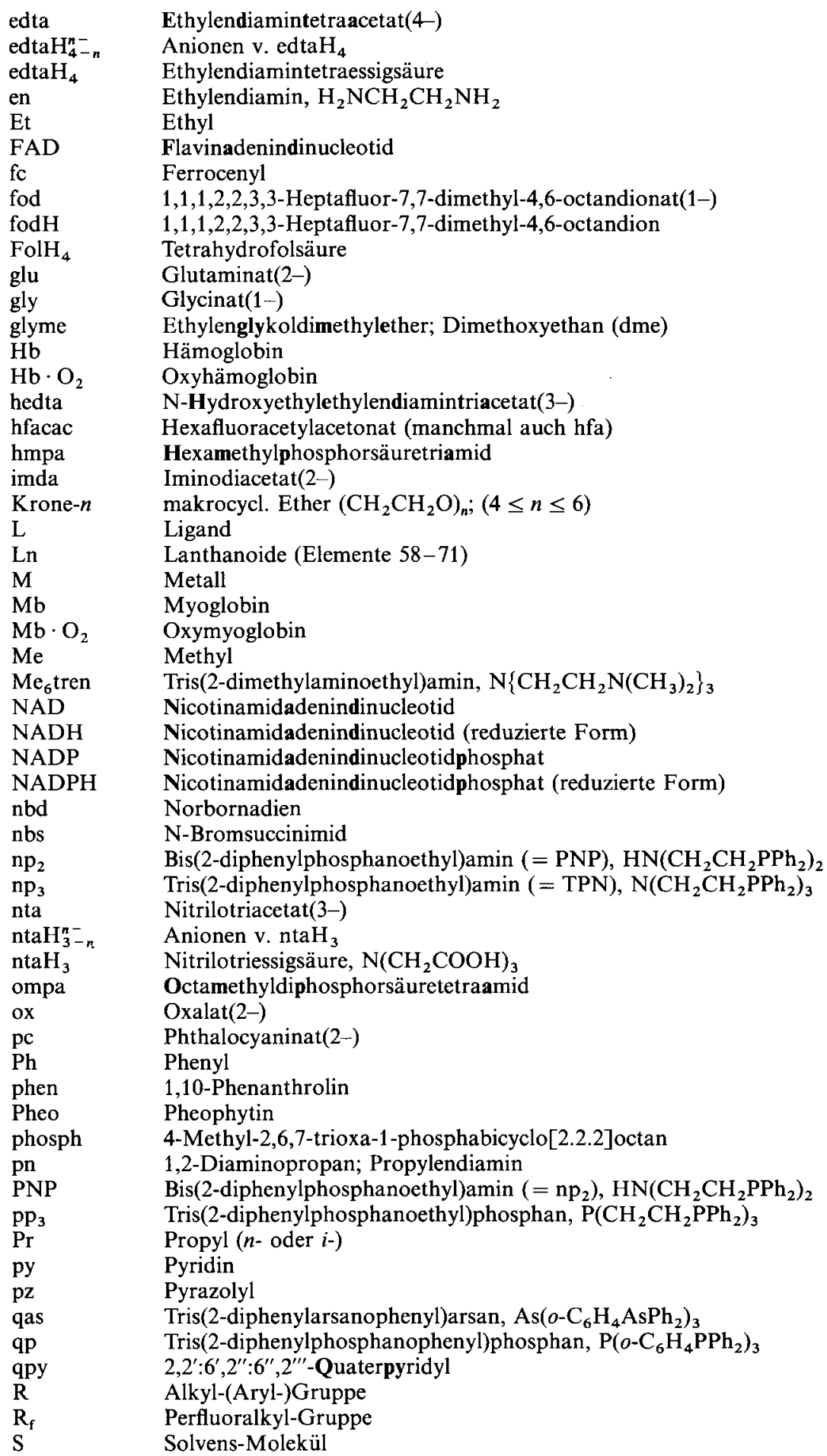




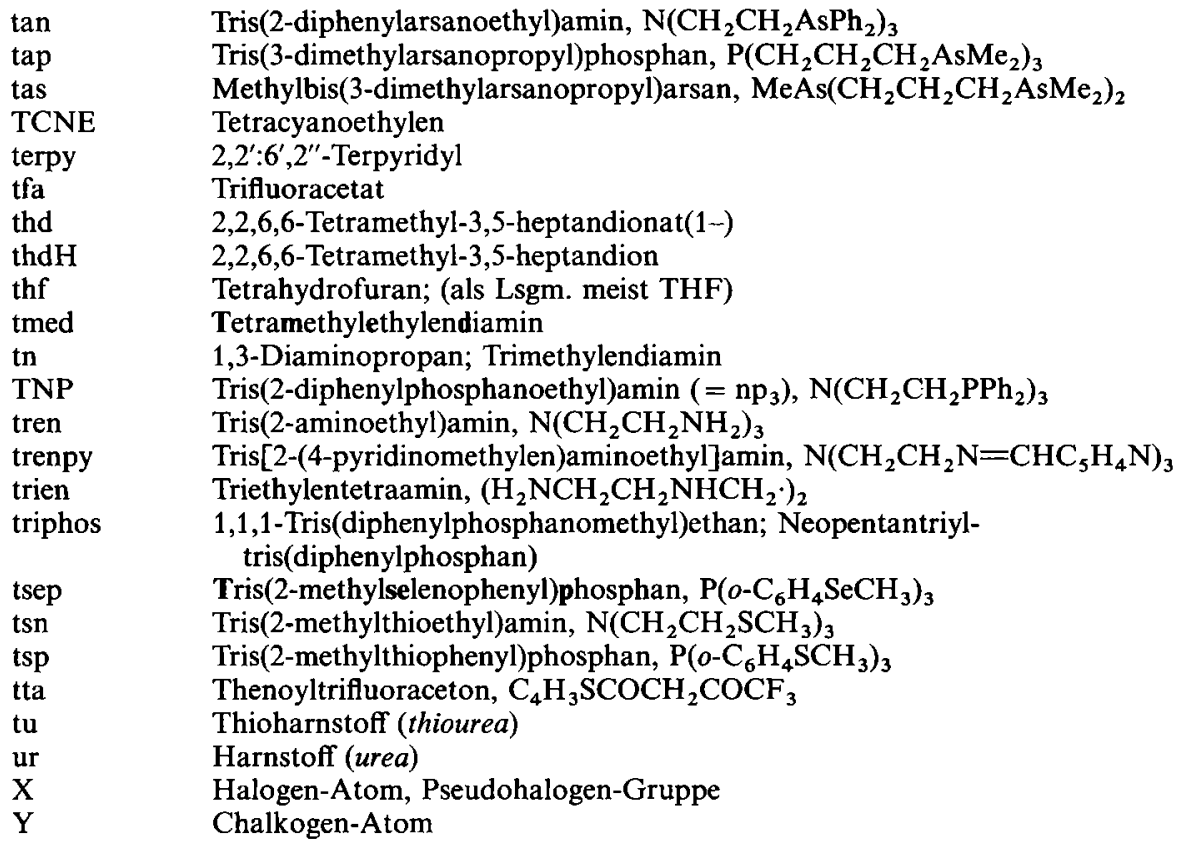


XXVIII Häufig gebrauchte Abkürzungen

\section{B Allgemeines}

AOM angular overlap-Methode, angular overlap-Modell

$\mathrm{AZ}$

B. M.

CB

$\mathrm{CD}$

CNO

CT

$D q$

$\mathrm{DZ}$

EA

EAN

$E M K$

ESCA

ESR

eu

$\mathrm{eV}$

EXAFS

$h d p$

HSAB

IE

IP

IR

IUPAC

$k d p$

$K_{\mathrm{L}}$

KTE

$\mathrm{KZ}$

LCAO

LF

LFSE

LGO

MO

MOSE

NMR

NQR

OPSE

ORD

PES

PS

PSEPT

QP

$q p$

SCF

SI

STE

TBP

$t b p$

UPS

UV

VB

VSEPR

XPS
Acceptorzahl

Bohrsche Magnetonen

korrespondierende Base (conjugate base)

Zirkulardichroismus

complete neglect of overlap

charge transfer

$\mathrm{Ma}$ f $\mathrm{f}$. Orbitalenergie-Aufspaltung im gerichteten Ligandenfeld

Donatorzahl

Elektronenaffinität

effektive Elektronenzahl (effective atomic number)

Elektromotorische Kraft $\mathscr{E}$

Elektronen-Spektroskopie zur chemischen Analyse

Elektronenspin-Resonanz

entropy units

Elektronvolt

extended $\boldsymbol{X}$-ray absorption fine structure

hexagonal dichteste Kugelpackung

harte und weiche Säuren und Basen (hard and soft acids and bases)

Ionisierungsenergie

Ionenpaar

Infrarot

Internationale Union für Reine und Angewandte Chemie (International Union of

Pure and Applied Chemistry)

kubisch dichteste Kugelpackung

Löslichkeitsprodukt

kinetischer trans-Effekt

Koordinationszahl

Linearkombination von Atomorbitalen

Ligandenfeld

Ligandenfeld-Stabilisierungsenergie

Ligandengruppen-Orbital

Molekülorbital

Molekülorbital-Stabilisierungsenergie

Kernmagnetische Resonanz

Kernquadrupol-Resonanz

Oktaederplatz-Stabilisierungsenergie

Optische Rotationsdispersion

Photoelektronen-Spektroskopie

Periodensystem

Polyeder-Skelett-Elektronenpaar-Theorie

quadratische Pyramide

quadratisch-pyramidal

self consistent field

Système International d'Unités

statischer trans-Effekt

trigonale Bipyramide

trigonal-bipyramidal

Ultraviolett-Photoelektronen-Spektroskopie, (manchmal auch UVPES)

Ultraviolett

Valenzstruktur (valence bond)

valence shell electron pair repulsion

Röntgen-Photoelektronen-Spektroskopie, (manchmal auch XPES) 